\title{
Plasmodium cynomolgi genome sequences provide insight into Plasmodium vivax and the monkey malaria clade
}

\author{
Shin-Ichiro Tachibana ${ }^{1,13}$, Steven A Sullivan ${ }^{2}$, Satoru Kawai ${ }^{3}$, Shota Nakamura ${ }^{4}$, Hyunjae R Kim ${ }^{2}$, \\ Naohisa Goto ${ }^{4}$, Nobuko Arisue ${ }^{5}$, Nirianne M Q Palacpac ${ }^{5}$, Hajime Honma ${ }^{1,5}$, Masanori Yagi ${ }^{5}$, Takahiro Tougan ${ }^{5}$, \\ Yuko Katakai ${ }^{6}$, Osamu Kaneko ${ }^{7}$, Toshihiro Mita ${ }^{8}$, Kiyoshi Kita ${ }^{9}$, Yasuhiro Yasutomi ${ }^{10}$, Patrick L Sutton ${ }^{2}$, \\ Rimma Shakhbatyan ${ }^{2}$, Toshihiro Horii ${ }^{5}$, Teruo Yasunaga ${ }^{4}$, John W Barnwell ${ }^{11}$, Ananias A Escalante ${ }^{12}$, \\ Jane M Carlton ${ }^{2,14} \&$ Kazuyuki Tanabe ${ }^{1,5,14}$
}

P. cynomolgi, a malaria-causing parasite of Asian Old World monkeys, is the sister taxon of $P$. vivax, the most prevalent malaria-causing species in humans outside of Africa. Because $P$. cynomolgi shares many phenotypic, biological and genetic characteristics with $P$. vivax, we generated draft genome sequences for three $P$. cynomolgi strains and performed genomic analysis comparing them with the $P$. vivax genome, as well as with the genome of a third previously sequenced simian parasite, Plasmodium knowlesi. Here, we show that genomes of the monkey malaria clade can be characterized by copy-number variants $(\mathrm{CNV})$ in multigene families involved in evasion of the human immune system and invasion of host erythrocytes. We identify genome-wide SNPs, microsatellites and CNVs in the $P$. cynomolgi genome, providing a map of genetic variation that can be used to map parasite traits and study parasite populations. The sequencing of the $P$. cynomolgi genome is a critical step in developing a model system for $P$. vivax research and in counteracting the neglect of $P$. vivax.

Human malaria is transmitted by anopheline mosquitoes and is caused by four species in the genus Plasmodium. Of these, P. vivax is the major malaria agent outside of Africa, annually causing 80-100 million cases ${ }^{1}$. Although $P$. vivax infection is often mistakenly regarded as benign and self-limiting, $P$. vivax treatment and control present challenges distinct from those of the more virulent Plasmodium falciparum. Biological traits, including a dormant (hypnozoite) liver stage responsible for recurrent infections (relapses), early infective sexual stages (gametocytes) and transmission from low parasite densities in the blood ${ }^{2}$, coupled with emerging antimalarial drug resistance ${ }^{3}$, render $P$. vivax resilient to modern control strategies. Recent evidence indicates that $P$. falciparum derives from parasites of great apes in Africa ${ }^{4}$, whereas P. vivax is more closely related to parasites of Asian Old World monkeys ${ }^{5-7}$, although not itself infective of these monkeys.

P. vivax cannot be cultured in vitro, and the small New World monkeys capable of hosting it are rare and do not provide an ideal model system. P. knowlesi, an Asian Old World monkey parasite recently recognized as a zoonosis for humans ${ }^{8}$, has had its genome sequenced ${ }^{9}$, but the species is distantly related to $P$. vivax and is phenotypically dissimilar. In contrast, $P$. cynomolgi, a simian parasite that can infect humans experimentally ${ }^{10}$, is the closest living relative (a sister taxon) to $P$. vivax and possesses most of the same genetic, phenotypic and biological characteristics-notably, periodic relapses caused by dormant hypnozoites, early infectious gametocyte formation and invasion of Duffy blood group-positive reticulocytes. P. cynomolgi thus offers a robust model for $P$. vivax in a readily available laboratory host, the Rhesus monkey, whose genome was recently sequenced ${ }^{11}$. Here, we report draft genome sequences of three $P$. cynomolgi strains and comparative genomic analyses of P. cynomolgi, P. vivax ${ }^{12}$ and $P$. knowlesi ${ }^{9}$, three members of the monkey malaria clade.

We sequenced the genome of P. cynomolgi strain B, isolated from a monkey in Malaysia and grown in splenectomized monkeys (Online Methods). A combination of Sanger, Roche 454 and Illumina chemistries was employed to generate a high-quality reference assembly at 161 -fold coverage, consisting of 14 supercontigs (corresponding to the 14 parasite chromosomes) and $\sim 1,649$ unassigned contigs, comprising

\footnotetext{
${ }^{1}$ Laboratory of Malariology, Research Institute for Microbial Diseases, Osaka University, Suita, Japan. ${ }^{2}$ Department of Biology, Center for Genomics and Systems Biology, New York University, New York, New York, USA. ${ }^{3}$ Laboratory of Tropical Medicine and Parasitology, Institute of International Education and Research, Dokkyo Medical University, Shimotsuga, Japan. ${ }^{4}$ Genome Information Research Center, Research Institute for Microbial Diseases, Osaka University, Suita, Japan. ${ }^{5}$ Department of Molecular Protozoology, Research Institute for Microbial Diseases, Osaka University, Suita, Japan. ${ }^{6}$ The Corporation for Production and Research of Laboratory Primates, Tsukuba, Japan. ${ }^{7}$ Department of Protozoology, Institute of Tropical Medicine (NEKKEN) and Global COE (Centers of Excellence) Program, Nagasaki University, Nagasaki, Japan. ${ }^{8}$ Department of Molecular and Cellular Parasitology, Graduate School of Medicine, Juntendo University, Tokyo, Japan. ${ }^{9}$ Department of Biomedical Chemistry, Graduate School of Medicine, The University of Tokyo, Tokyo, Japan. ${ }^{10}$ Tsukuba Primate Research Center, National Institute of Biomedical Innovation, Tsukuba, Japan. ${ }^{11}$ Center for Global Health, Centers for Disease Control and Prevention, Divison of Parasitic Diseases and Malaria, Atlanta, Georgia, USA. ${ }^{12}$ Center for Evolutionary Medicine and Informatics, The Biodesign Institute, Arizona State University, Tempe, Arizona, USA. ${ }^{13}$ Present address: Career-Path Promotion Unit for Young Life Scientists, Kyoto University, Kyoto, Japan. ${ }^{14}$ These authors jointly directed this work. Correspondence should be addressed to K.T. (kztanabe@biken.osaka-u.ac.jp) or J.M.C. (jane.carlton@nyu.edu).
} 
a total length of $\sim 26.2 \mathrm{Mb}$ (Supplementary Table 1). Comparing genomic features of $P$. cynomolgi, $P$. knowlesi and $P$. vivax reveals many similarities, including GC content (mean GC content of $40.5 \%$ ), 14 positionally conserved centromeres and the presence of intrachromosomal telomeric sequences (ITSs; GGGTT(T/C)A), which were discovered in the P. knowlesi genome ${ }^{9}$ but are absent in P. vivax (Fig. 1, Table 1 and Supplementary Table 2).

We annotated the $P$. cynomolgi strain B genome using a combination of $a b$ initio gene prediction programs trained on high-quality data sets and sequence similarity searches against the annotated $P$. vivax and P. knowlesi genomes. Not unexpectedly for species from the same monkey malaria clade, gene synteny along the 14 chromosomes is highly conserved, although numerous microsyntenic breaks are present in regions containing multigene families (Fig. 2 and Table 2). This genome-wide view of synteny in six species of Plasmodium also identified two apparent errors in existing public sequence databases: an inversion in chromosome 3 of $P$. knowlesi and an inversion in chromosome 6 of $P$. vivax. The $P$. cynomolgi genome contains 5,722 genes, of which approximately half encode conserved hypothetical proteins of unknown function, as is the case in all the Plasmodium genomes sequenced to date. A maximum-likelihood phylogenetic tree constructed using 192 conserved ribosomal and translation- and transcription-related genes (Supplementary Fig. 1) confirms the close relationship of P. cynomolgi to P. vivax compared to five other Plasmodium species. Approximately $90 \%$ of genes $(4,613)$ have reciprocal best-match orthologs in all three species (Fig. 3), enabling refinement of the existing $P$. vivax and P. knowlesi annotations (Supplementary Table 3). The high degree of gene orthology enabled us to identify specific examples of gene duplication (an important vehicle for genome evolution), including a duplicated homolog of $P$. vivax Pvs28-which encodes a sexual stage surface antigen that is a transmission-blocking vaccine candidate $^{13}$-in P. cynomolgi (Supplementary Table 4). Genes common only to $P$. cynomolgi and $P$. vivax $(n=214)$ outnumber those that are restricted to P. cynomolgi and P. knowlesi $(n=100)$ or $P$. vivax and $P$. knowlesi $(n=17)$. Such figures establish the usefulness of $P$. cynomolgi as a model species for studying the more intractable $P$. vivax.

Figure 1 Architecture of the $P$. cynomolgi genome and associated genomewide variation data. Data are shown for each of the 14 P. cynomolgi chromosomes. The six concentric rings, from outermost to innermost, represent (i) the location of 5,049 P. cynomolgi genes, excluding those on small contigs (cyan lines); (ii) genome features, including 14 centromeres (thick black lines), 43 telomeric sequence repeats (short red lines), 43 tRNA genes (red lines), 10 rRNAs (dark blue lines) and several gene family members, including 53 cyir (dark green lines), 8 rbp (brown lines), 13 sera (serine-rich antigen; pink lines), 25 trag (tryptophan-rich antigen; purple lines), 12 msp3 (merozoite surface protein 3; light gray lines), $13 \mathrm{msp} 7$ (merozoite surface protein 7; gray lines), $25 \mathrm{rad}$ (silver lines), 8 etramp (orange lines), 16 Pf-fam-b (light blue lines) and 7 Pv-fam- $d$ (light green lines); (iii) plot of $d_{S} / d_{N}$ for 4,605 orthologs depicting genome-wide polymorphism within P. cynomolgi strains B and Berok (black line) and divergence between P. cynomolgi strains B and Berok and $P$. vivax Salvador I (red line); a track above the plot indicates $P$. cynomolgi genes under positive selection (red) and purifying selection (blue), and a track below the plot indicates $P$. cynomolgi-P. vivax orthologs under positive selection (red) and purifying selection (blue); (iv) heatmap indicating SNP density of 3 P. cynomolgi strains plotted per 10-kb windows: red, 0-83 SNPs per $10 \mathrm{~kb}$ (regions of lowest SNP density); blue, 84-166 SNPs per $10 \mathrm{~kb}$; green, 167-250 SNPs per $10 \mathrm{~kb}$; purple, 251-333 SNPs per $10 \mathrm{~kb}$; orange, 334-416 SNPs per10 kb; yellow,

417-500 SNPs per10 kb (regions of highest SNP density); (v) $\log _{2}$ ratio plot of CNVs identified from a comparison of $P$. cynomolgi strains B and Berok; and (vi) map of 182 polymorphic intergenic microsatellites (MS, black dots). The figure was generated using Circos software (see URLs).

Notably, most of the genes specific to a particular species belong to multigene families (excluding hypothetical genes; Table 2 and Supplementary Table 5). This suggests repeated lineage-specific gene duplication and/or gene deletion in multigene families within the three monkey malaria clade species. Moreover, copy numbers of the genes composing multigene families were generally greater in the $P$. cynomolgi-P. vivax lineage than in $P$. knowlesi, suggesting repeated gene duplication in the ancestral lineage of $P$. cynomolgi and $P$. vivax (or repeated gene deletion in the $P$. knowlesi lineage). Thus, the genomes of P. cynomolgi, P. vivax and P. knowlesi can largely be distinguished by variations in the copy number of multigene family members. Examples of such families include those that encode proteins involved in evasion of the human immune system (vir, kir and SICAvar) and invasion of host red blood cells ( $d b p$ and $r b p)$.

In malaria-causing parasites, invasion of host erythrocytes, mediated by specific interactions between parasite ligands and erythrocyte receptors, is a crucial component of the parasite lifecycle. Of great interest are the $e b l$ and $r b l$ gene families, which encode parasite ligands required for the recognition of host erythrocytes. The $e b l$ genes encode erythrocyte binding-like (EBL) ligands such as the Duffy-binding proteins (DBPs) that bind to Duffy antigen receptor for chemokines (DARC) on human and monkey erythrocytes. The $r b l$ genes encode the reticulocyte binding-like (RBL) protein family, including reticulocyte-binding proteins (RBPs) in P. cynomolgi and P. vivax, and normocyte-binding proteins (NBPs) in P. knowlesi, which bind to unknown erythrocyte receptors $^{14}$. We confirmed the presence of two $d b p$ genes in P. cynomolg $^{15}$ (Supplementary Table 6), in contrast to the one $d b p$ and three $d b p$ genes identified in $P$. vivax and $P$. knowlesi, respectively. This raises an intriguing hypothesis that $P$. vivax lost one $d b p$ gene, and thus its infectivity of Old World monkey erythrocytes, after divergence from a common P. vivax-P. cynomolgi ancestor. This hypothesis is also supported by our identification of single-copy $d b p$ genes in two other closely related Old World monkey malaria-causing parasites, Plasmodium fieldi and Plasmodium simiovale, which are incapable of infecting humans ${ }^{16}$. These two Old World monkey species lost one or more $d b p$ genes during divergence that confer infectivity to humans, whereas P. cynomolgi and P. knowlesi retained $d b p$ genes that allow invasion of human erythrocytes (Supplementary Fig. 2).

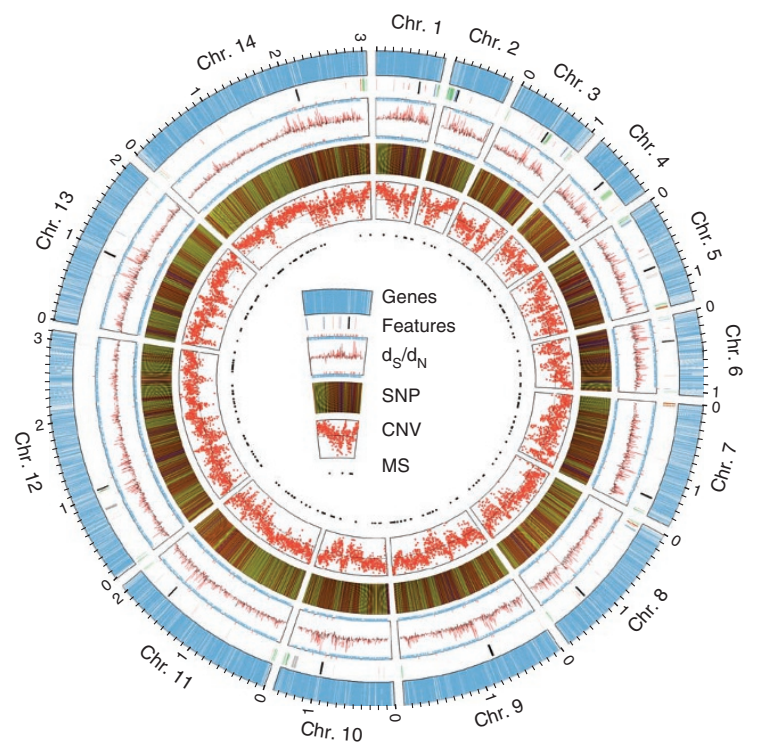


Table 1 Comparison of genome features between $P$. cynomolgi, $P$. vivax and $P$. knowlesi, three species of the monkey malaria clade

\begin{tabular}{|c|c|c|c|}
\hline Feature & P. cynomolgi & P. vivax $^{12}$ & P. knowlesi ${ }^{9}$ \\
\hline \multicolumn{4}{|l|}{ Assembly } \\
\hline Size (Mb) & 26.2 & 26.9 & 23.7 \\
\hline $\begin{array}{l}\text { Number of } \\
\text { scaffolds }\end{array}$ & $14(1,649)$ & $14(2,547)$ & $14(67)$ \\
\hline Coverage (fold) & 161 & 10 & 8 \\
\hline GC content (\%) & 40.4 & 42.3 & 38.8 \\
\hline \multicolumn{4}{|l|}{ Genes } \\
\hline Number of genes & 5,722 & 5,432 & 5,197 \\
\hline $\begin{array}{l}\text { Mean gene length } \\
\text { (bp) }\end{array}$ & 2,240 & 2,164 & 2,180 \\
\hline $\begin{array}{l}\text { Gene density } \\
\quad{\text { (bp per gene })^{b}}^{\text {b }}\end{array}$ & $4,428.2$ & $4,950.5$ & $4,416.1$ \\
\hline $\begin{array}{l}\text { Percentage } \\
\text { codingb }\end{array}$ & 51.0 & 47.1 & 49.0 \\
\hline \multicolumn{4}{|l|}{ Structural RNAs } \\
\hline $\begin{array}{l}\text { Number of tRNA } \\
\text { genes }\end{array}$ & 43 & 44 & 41 \\
\hline $\begin{array}{l}\text { Number of } 5 S \\
\text { rRNA genes }\end{array}$ & 3 & 3 & $\mathrm{O}^{\mathrm{c}}$ \\
\hline $\begin{array}{c}\text { Number of } 5.8 S \\
18 S \text { and } 28 S \\
\text { rRNA units }\end{array}$ & 7 & 7 & 5 \\
\hline \multicolumn{4}{|l|}{ Nuclear genome } \\
\hline $\begin{array}{l}\text { Number of } \\
\text { chromosomes }\end{array}$ & 14 & 14 & 14 \\
\hline $\begin{array}{l}\text { Number of } \\
\text { centromeres }\end{array}$ & 14 & 14 & 14 \\
\hline Isochore structure ${ }^{d}$ & + & + & - \\
\hline \multicolumn{4}{|l|}{ Mitochondrial genome } \\
\hline Size $(b p)^{e}$ & $5,986(A B 444123)$ & 5,990 (AY59814 & 5,958 (AB444108) \\
\hline GC content ( $\%)$ & 30.3 & 30.5 & 30.5 \\
\hline \multicolumn{4}{|l|}{ Apicoplast genome } \\
\hline Size (bp) & $29,297^{f}$ & $5,064^{g}$ & $\mathrm{~N} / \mathrm{A}$ \\
\hline GC content ( $\%)$ & 13.0 & 17.1 & $\mathrm{~N} / \mathrm{A}$ \\
\hline
\end{tabular}

$\mathrm{N} / \mathrm{A}$, not available.

aSmall unassigned contigs indicated in parentheses. bSequence gaps excluded. cNot present in P. knowlesi assembly version 4.0 . dRegions of the genome that differ in density and are separable by $\mathrm{CsCl}$ centrifugation; isochores correspond to domains differing in $\mathrm{GC}$ content. eldentified in other studies (GenBank accessions given in parentheses). ${ }^{\mathrm{f}} \mathrm{Partial}$ sequence ( $\sim 86 \%$ complete) generated during this project. gPartial sequence of reference genome only published ${ }^{12}$, actual size is $\sim 35 \mathrm{~kb}$.

We found multiple rbp genes, some truncated or present as pseudogenes, in the P. cynomolgi genome (Fig. 1 and Table 2). Phylogenetic analysis showed that $r b l$ genes from $P$. cynomolgi, $P$. vivax and $P$. knowlesi can be classified into three distinct groups, RBP/NBP-1, RBP/NBP-2 and RBP/NBP-3 (Supplementary Fig. 3), and suggests that these groups existed before the three species diverged. All three groups of RBP/NBP are represented in P. cynomolgi, whereas $P$. vivax and $P$. knowlesi lack functional genes from the RBP/NBP-3 and RBP/ NBP-1 groups, respectively. Thus, $r b l$ gene family expansion seems to have occurred after speciation, indicating that the three species have multiple species-specific erythrocyte invasion mechanisms. Notably, we found an ortholog of $P$. vivax rbp $1 b$ in some strains of $P$. cynomolgi but not in others (Supplementary Table 6). To our knowledge, this

Figure 2 Genome synteny between six species of Plasmodium parasite. Protein-coding genes of $P$. cynomolgi are shown aligned with those of five other Plasmodium genomes: two species belonging to the monkey malaria clade, $P$. vivax and $P$. knowlesi; two species of rodent malaria, $P$. berghei and $P$. chabaudi; and $P$. falciparum. Highly conserved proteincoding regions between the genomes are colored in order from red $\left(5^{\prime}\right.$ end of chromosome 1 ) to blue ( $3^{\prime}$ end of chromosome 14 ) with respect to genomic position of $P$. cynomolgi. is the first example of a $\mathrm{CNV}$ for a $r b p$ gene between strains of a single Plasmodium species, highlighting how repeated creation and destruction of $r b l$ genes, a signature of adaptive evolution, may have enabled species of the monkey malaria clade to expand or switch between monkey and human hosts.

The largest gene family in P. cynomolgi, consisting of 256 cyir (cynomolgi-interspersed repeat) genes, is part of the pir (plasmodiuminterspersed repeat) superfamily that includes $P$. vivax vir genes $(n=319)$ and $P$. knowlesi kir genes $(n=70)$ (Table 2). Pir-encoded proteins reside on the surface of infected erythrocytes and have an important role in immune evasion ${ }^{17}$. Most cyir genes have sequence similarity to $P$. vivax vir genes $(n=254$; Supplementary Table 7$)$ and are found in subtelomeric regions (Fig. 1), but, notably, 11 cyir genes have sequence similarity to $P$. knowlesi kir genes (Supplementary Table 7) and occur more internally in the chromosomes, as do the kir genes in P. knowlesi. As with 'molecular mimicry' in P. knowlesi (mimicry of host sequences by pathogen sequences) ${ }^{9}$, one CYIR protein (encoded by PCYB_032250) has a region of 56 amino acids that is highly similar to the extracellular domain of primate CD99 (Supplementary Fig. 4), a molecule involved in the regulation of T-cell function. A new finding is that P. cynomolgi has two genes whose sequences are similar to $P$. knowlesi SICAvar genes (Supplementary Table 7) that are expressed on the surfaces of schizont-infected macaque erythrocytes and are involved in antigenic variation ${ }^{18}$.

The ability to form a dormant hypnozoite stage is common to both $P$. cynomolgi and $P$. vivax and was first shown in laboratory infections of monkeys by mosquito-transmitted $P$. cynomolgi ${ }^{19}$. In a search for candidate genes involved in the hypnozoite stage, we identified nine coding for 'dormancy-related' proteins that had the upstream ApiAP2 motifs ${ }^{20}$ necessary for stage-specific transcriptional regulation at the sporozoite (pre-hypnozoite) stage (Supplementary Table 8). The candidates include kinases that are involved in cell cycle transition; hypnozoite formation may be regulated by phosphorylation of proteins specifically expressed at the pre-hypnozoite stage. Our list of $P$. cynomolgi candidate genes represents an informed starting point for experimental studies of this elusive stage.

We sequenced P. cynomolgi strains Berok (from Malaysia) and Cambodian (from Cambodia) to $26 \times$ and $17 \times$ coverage, respectively, to characterize $P$. cynomolgi genome-wide diversity through analysis of SNPs, CNVs and microsatellites. A comparison of the three P. cynomolgi strains identified 178,732 SNPs (Supplementary Table 9) at a frequency of 1 SNP per $151 \mathrm{bp}$, a polymorphism level somewhat

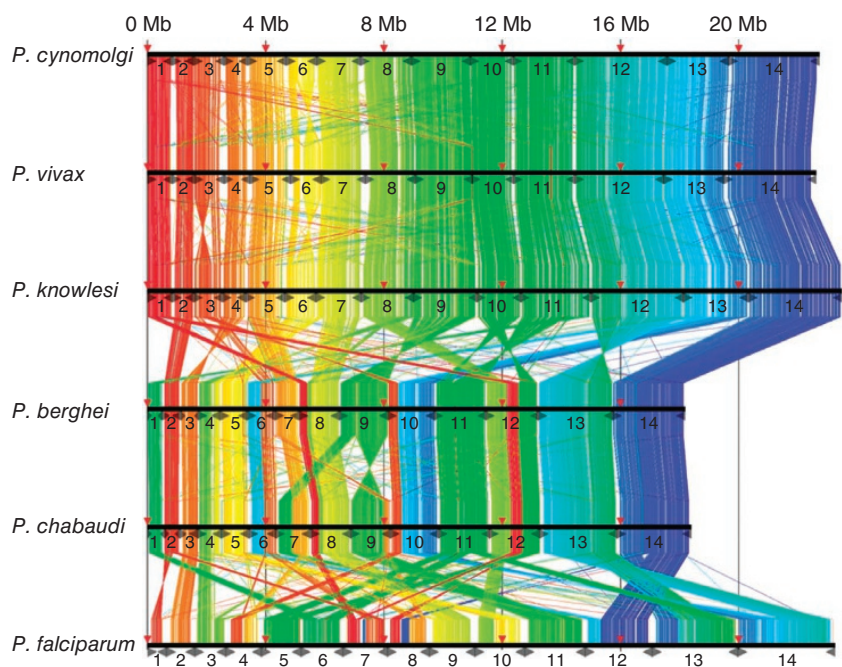


Table 2 Components of multigene families of $P$. cynomolgi, $P$. vivax and $P$. knowlesi differ in copy number

\begin{tabular}{|c|c|c|c|c|c|c|c|}
\hline Family & Multigene family & Localization & Arrangement & P. cynomolgi & P. vivax & P. knowlesi & Putative function and other information \\
\hline 1 & pir (vir-like) & Subtelomeric & Scattered and clustered & 254 & $319^{a}$ & 4 & Immune evasion \\
\hline 2 & pir (kir-like) & Subtelomeric and central & Scattered and clustered & 11 & 2 & $66^{a}$ & Immune evasion \\
\hline 3 & SICAvar & Subtelomeric and central & Scattered and clustered & 2 & 1 & $242^{a}$ & Antigenic variation, immune evasion \\
\hline 4 & $m s p 3$ & Central & Clustered & 12 & 12 & 3 & Merozoite surface protein \\
\hline 5 & $m s p 7$ & Central & Clustered & 13 & 13 & 5 & Merozoite surface protein \\
\hline 6 & $d b /(d b p / e b /)$ & Subtelomeric & Scattered & 2 & 1 & 3 & Host cell recognition \\
\hline 7 & $r b /(r b p / n b p / r h)$ & Subtelomeric & Scattered & $8^{a}$ & $10^{\mathrm{a}}$ & $3^{a}$ & Host cell recognition \\
\hline 8 & Pv-fam-a (trag) & Subtelomeric & Scattered and clustered & 36 & 36 & $26^{a}$ & Tryptophan-rich \\
\hline 9 & $P v-f a m-b$ & Central & Clustered & 3 & 6 & 1 & Unknown \\
\hline 10 & $P v-f a m-c$ & Subtelomeric & Unknown ${ }^{b}$ & 1 & 7 & 0 & Unknown \\
\hline 11 & Pv-fam-d (hypb) & Subtelomeric & Scattered & 18 & 16 & 2 & Unknown \\
\hline 12 & Pv-fam-e (rad) & Subtelomeric & Clustered & 27 & 44 & 16 & Unknown \\
\hline 13 & $P v$-fam-g & Central & Clustered & 3 & 3 & 3 & Unknown \\
\hline 14 & Pv-fam-h (hyp16) & Central & Clustered & 6 & 4 & 2 & Unknown \\
\hline 15 & $P v-f a m-i(h y p 11)$ & Subtelomeric & Scattered & 6 & 6 & 5 & Unknown \\
\hline 16 & Pk-fam-a & Central & Scattered & 0 & 0 & $12^{\mathrm{a}}$ & Unknown \\
\hline 17 & $P k-f a m-b$ & Subtelomeric & Scattered & 0 & 0 & 9 & Unknown \\
\hline 18 & $P k-f a m-c$ & Subtelomeric & Scattered & 0 & 0 & $6^{\mathrm{a}}$ & Unknown \\
\hline 19 & Pk-fam-d & Central & Scattered & 0 & 0 & $3^{a}$ & Unknown \\
\hline 20 & Pk-fam-e & Subtelomeric & Scattered & 0 & 0 & $3^{a}$ & Unknown \\
\hline 21 & PST-A & Subtelomeric and central & Scattered & $9^{a}$ & $11^{\mathrm{a}}$ & 7 & $\alpha \beta$ hydrolase \\
\hline 22 & ETRAMP & Subtelomeric & Scattered & 9 & 9 & 9 & Parasitophorous vacuole membrane \\
\hline 23 & CLAG (RhopH-1) & Subtelomeric & Scattered & 2 & 3 & 2 & $\begin{array}{l}\text { High-molecular-wieght rhoptry antigen } \\
\text { complex }\end{array}$ \\
\hline 24 & PVSTP1 & Subtelomeric & Unknown ${ }^{b}$ & 3 & $10^{\mathrm{a}}$ & 0 & Unknown \\
\hline 25 & PHIST (Pf-fam-b) & Subtelomeric & Scattered and clustered & 21 & 20 & 15 & Unknown \\
\hline 26 & SERA & Central & Clustered & $13^{a}$ & $13^{a}$ & $8^{a}$ & Cysteine protease \\
\hline
\end{tabular}

aPseudogenes, truncated genes and gene fragments included. ${ }^{b}$ Gene arrangement could not be determined due to localization on unassigned contigs.

similar to that found when P. falciparum genomes are compared ${ }^{21,22}$. We calculated the pairwise nucleotide diversity $(\pi)$ as $5.41 \times 10^{-3}$ across the genome, which varies little between the chromosomes. We assessed genome-wide CNVs between the P. cynomolgi B and Berok strains, using a robust statistical model in the CNV-seq program $^{23}$, by which we identified $1,570 \mathrm{CNVs}$ (1 per $17 \mathrm{~kb}$ ), including 1 containing the $r b p 1 b$ gene on chromosome 7 (Supplementary Fig. 5). Finally, mining of the P. cynomolgi B and Berok strains identified 182 polymorphic intergenic microsatellites (Supplementary Table 10), the first set of genetic markers developed for this species. These provide a toolkit for studies of genetic diversity and population structure of laboratory stocks or natural infections of P. cynomolgi, many of which have recently been isolated from screening hundreds of wild monkeys for the zoonosis P. knowlesi ${ }^{24}$.

We estimated the difference between the number of synonymous changes per synonymous site $\left(d_{S}\right)$ and the number of nonsynonymous changes per nonsynonymous site $\left(\mathrm{d}_{\mathrm{N}}\right)$ over 4,563 pairs of orthologs within P. cynomolgi strains B and Berok and 4,601 pairs of orthologs between these two P. cynomolgi strains and P. vivax Salvador I, using a simple Nei-Gojobori model ${ }^{25}$. We found 63 genes with $\mathrm{d}_{\mathrm{N}}>\mathrm{d}_{\mathrm{S}}$ within the two $P$. cynomolgi strains and 3,265 genes with $d_{S}>d_{N}$ (Supplementary Table 11). Genes with relatively high $d_{N} / d_{S}$ ratios include those encoding transmembrane proteins, such as antigens and transporters, among which is a transmission-blocking target antigen, Pcyn230 (encoded by PCYB_042090). Notably, the P. vivax ortholog (PVX_003905) does not show evidence for positive selection ${ }^{26}$, suggesting species-specific positive selection. We explored the degree to which evolution of orthologs has been constrained between P. cynomolgi and P. vivax and found 83 genes under possible accelerated evolution but 3,739 genes under possible purifying selection (Supplementary Table 12). This conservative estimate indicates that at least $81 \%$ of loci have diverged under strong constraint, compared with $1.8 \%$ of loci under less constraint or positive selection (Fig. 1), indicating that, overall, the genome of $P$. cynomolgi is highly conserved in single-locus genes compared to $P$. vivax and emphasizing the value of $P$. cynomolgi as a biomedical and evolutionary model for studying $P$. vivax.

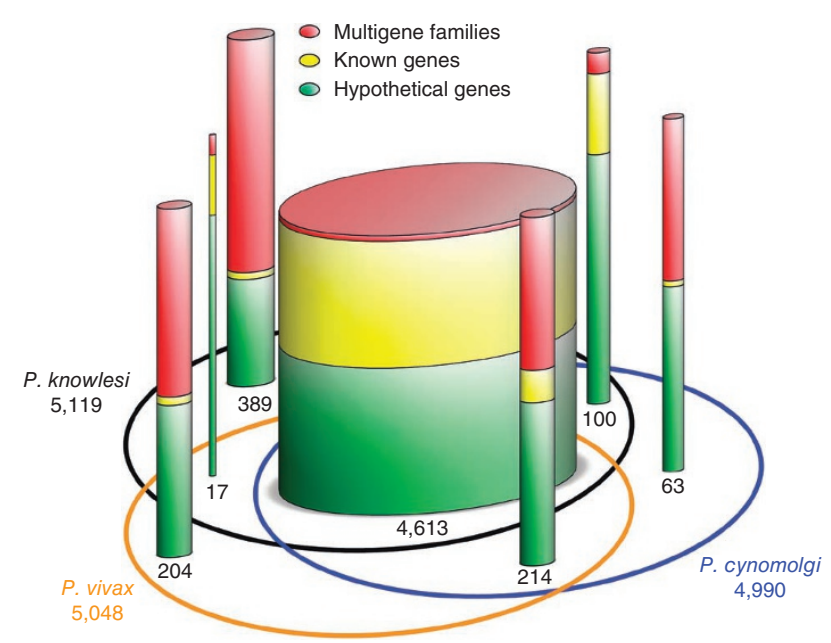

Figure 3 Comparison of the genes of P. cynomolgi, P. vivax and $P$. knowlesi. The Venn ellipses represent the three genomes, with the total number of genes assigned to the chromosomes indicated under the species name. Cylinders depict orthologous and non-orthologous genes between the three genomes, with the number of genes in each indicated and represented graphically by cylinder relative width. In each cylinder, genes are divided into three categories whose thickness is represented by colored bands proportional to category percentage. 
Our generation of the first $P$. cynomolgi genome sequences is a critical step in the development of a robust model system for the intractable and neglected $P$. vivax species ${ }^{27}$. Comparative genome analysis of $P$. vivax and the Old World monkey malaria-causing parasites $P$. cynomolgi and $P$. knowlesi presented here provides the foundation for further insights into traits such as host specificity that will enhance prospects for the eventual elimination of vivax-caused malaria and global malaria eradication.

URLs. PlasmoDB, http://plasmodb.org/; Circos, http://circos.ca/; MIcroSAtelite Identification tool (MISA), http://pgrc.ipk-gatersleben. de/misa/; dbSNP, http://www.ncbi.nlm.nih.gov/projects/SNP/snp_ viewBatch.cgi? sbid=1056645.

\section{METHODS}

Methods and any associated references are available in the online version of the paper.

Accession codes. Sequence data for the P. cynomolgi B, Cambodian and Berok strains have been deposited in the DNA Data Bank of Japan (DDBJ), the European Molecular Biology Laboratory (EMBL) and the GenBank databases under the following accessions: B strain sequence reads DRA000196, genome assembly BAEJ01000001-BAEJ01003341 and annotation DF157093-DF158755; Cambodian strain sequence reads DRA000197; and Berok strain sequence reads SRA047950. SNP calls have been submitted to dbSNP (NYU_CGSB_BIO; 1056645) and may also be downloaded from the dbSNP website (see URLs). Sequences of the $d b p$ genes from P. cynomolgi (Cambodian strain), P. fieldi (A.b.i. strain) and $P$. simiovale (AB617788-AB617791) and the P. cynomolgi Berok strain (JQ422035-JQ422036) and $r b p$ gene sequences from the P. cynomolgi Berok and Cambodian strains (JQ422037-JQ422050) have been deposited. A partial apicoplast genome of the P. cynomolgi Berok strain has been deposited (JQ522954). The P. cynomolgi B reference genome is also available through PlasmoDB (see URLs).

Note: Supplementary information is available in the online version of the paper.

\section{ACKNOWLEDGMENTS}

We thank H. Sawai for suggestions on genome analysis, D. Fisher for help with genome-wide evolutionary analyses and the NYU Langone Medical Center Genome Technology Core for access to Roche 454 sequencing equipment (funded by grant S10 RR026950 to J.M.C. from the US National Institutes of Health (NIH)). Genome and phylogenetic analyses used the Genome Information Research Center in the Research Institute of Microbial Diseases at Osaka University. This work was supported by grants from the Ministry of Education, Culture, Sports, Science and Technology of Japan (18073013, 18GS03140013, 20390120 and 22406012) to K.T., an NIH grant (R01 GM080586) to A.A.E. and a Burroughs Wellcome Fund grant (1007398) and an NIH International Centers of Excellence for Malaria Research grant (U19 AI089676-01) to J.M.C. The content is soley the responsibility of the authors and does not necessarily represent the official views of the NIH.

\section{AUTHOR CONTRIBUTIONS}

K.T., J.M.C., A.A.E. and J.W.B. conceived and conducted the study. S.K., Y.K., Y.Y., S.-I.T. and J.W.B. provided P. cynomolgi material. S.N., N.G., T.Y. and H.R.K. constructed a computing system for data processing, and S.-I.T., H.H., P.L.S., S.A.S. and H.R.K. performed scaffolding of contigs and manual annotation of the predicted genes. S.N. performed sequence correction of supercontigs and gene prediction. S.-I.T., S.N., N.G., N.A., M.Y., O.K., K.T., H.R.K., R.S., S.A.S. and J.M.C. analyzed data. S.-I.T., N.M.Q.P., T.T., T.M., K.K., J.M.C., T.H., A.A.E., J.W.B. and K.T. wrote the manuscript.

\section{COMPETING FINANCIAL INTERESTS}

The authors declare no competing financial interests.

Published online at http://www.nature.com/doifinder/10.1038/ng.2375.

Reprints and permissions information is available online at http://www.nature.com/ reprints/index.html.

This work is licensed under a Creative Commons Attribution-NonCommercialShareAlike 3.0 Unported (CC BY-NC-SA) license. To view a copy of this license, visit http://creativecommons.org/licenses/by-nc-sa/3.0/.

1. Mendis, K., Sina, B.J., Marchesini, P. \& Carter, R. The neglected burden of Plasmodium vivax malaria. Am. J. Trop. Med. Hyg. 64, 97-106 (2001).

2. Mueller, I. et al. Key gaps in the knowledge of Plasmodium vivax, a neglected human malaria parasite. Lancet Infect. Dis. 9, 555-566 (2009).

3. Baird, J.K. Resistance to chloroquine unhinges vivax malaria therapeutics Antimicrob. Agents Chemother. 55, 1827-1830 (2011).

4. Rayner, J.C., Liu, W., Peeters, M., Sharp, P.M. \& Hahn, B.H. A plethora of Plasmodium species in wild apes: a source of human infection? Trends Parasitol. 27, 222-229 (2011).

5. Cornejo, O.E. \& Escalante, A.A. The origin and age of Plasmodium vivax. Trends Parasitol. 22, 558-563 (2006).

6. Escalante, A.A. et al. A monkey's tale: the origin of Plasmodium vivax as a human malaria parasite. Proc. Natl. Acad. Sci. USA 102, 1980-1985 (2005).

7. Mu, J. et al. Host switch leads to emergence of Plasmodium vivax malaria in humans. Mol. Biol. Evol. 22, 1686-1693 (2005).

8. Singh, B. et al. A large focus of naturally acquired Plasmodium knowlesi infections in human beings. Lancet 363, 1017-1024 (2004).

9. Pain, A. et al. The genome of the simian and human malaria parasite Plasmodium knowlesi. Nature 455, 799-803 (2008).

10. Eyles, D.E., Coatney, G.R. \& Getz, M.E. Vivax-type malaria parasite of macaques transmissible to man. Science 131, 1812-1813 (1960).

11. Gibbs, R.A. et al. Evolutionary and biomedical insights from the rhesus macaque genome. Science 316, 222-234 (2007).

12. Carlton, J.M. et al. Comparative genomics of the neglected human malaria parasite Plasmodium vivax. Nature 455, 757-763 (2008).

13. Saxena, A.K., Wu, Y. \& Garboczi, D.N. Plasmodium p25 and p28 surface proteins: potential transmission-blocking vaccines. Eukaryot. Cell 6, 1260-1265 (2007).

14. Iyer, J., Gruner, A.C., Renia, L., Snounou, G. \& Preiser, P.R. Invasion of host cells by malaria parasites: a tale of two protein families. Mol. Microbiol. 65, 231-249 (2007).

15. Okenu, D.M., Malhotra, P., Lalitha, P.V., Chitnis, C.E. \& Chauhan, V.S. Cloning and sequence analysis of a gene encoding an erythrocyte binding protein from Plasmodium cynomolgi. Mol. Biochem. Parasitol. 89, 301-306 (1997).

16. Coatney, G.R., Collins, W.E., Warren, M. \& Contacos, P.G. The Primate Malarias (US Department of Health, Education and Welfare, Washington, DC, 1971).

17. Cunningham, D., Lawton, J., Jarra, W., Preiser, P. \& Langhorne, J. The pir multigene family of Plasmodium: antigenic variation and beyond. Mol. Biochem. Parasitol. 170, 65-73 (2010).

18. al-Khedery, B., Barnwell, J.W. \& Galinski, M.R. Antigenic variation in malaria: a 3 genomic alteration associated with the expression of a $P$. knowlesi variant antigen. Mol. Cell 3, 131-141 (1999).

19. Krotoski, W.A. The hypnozoite and malarial relapse. Prog. Clin. Parasitol. 1, 1-19 (1989).

20. Campbell, T.L., De Silva, E.K. Olszewski, K.L., Elemento, O \& Llinas, M. Identification and genome-wide prediction of DNA binding specificities for the ApiAP2 family of regulators from the malaria parasite. PLoS Pathog. 6, e1001165 (2010).

21. Mu, J. et al. Genome-wide variation and identification of vaccine targets in the Plasmodium falciparum genome. Nat. Genet. 39, 126-130 (2007).

22. Volkman, S.K. et al. A genome-wide map of diversity in Plasmodium falciparum. Nat. Genet. 39, 113-119 (2007).

23. Xie, C. \& Tammi, M.T. CNV-seq, a new method to detect copy number variation using high-throughput sequencing. BMC Bioinformatics 10, 80 (2009).

24. Lee, K.S. et al. Plasmodium knowlesi: reservoir hosts and tracking the emergence in humans and macaques. PLoS Pathog. 7, e1002015 (2011).

25. Nei, M. \& Gojobori, T. Simple methods for estimating the numbers of synonymous and nonsynonymous nucleotide substitutions. Mol. Biol. Evol. 3, 418-426 (1986).

26. Doi, M. et al. Worldwide sequence conservation of transmission-blocking vaccine candidate Pvs230 in Plasmodium vivax. Vaccine 29, 4308-4315 (2011).

27. Carlton, J.M., Sina, B.J. \& Adams, J.H. Why is Plasmodium vivax a neglected tropical disease? PLoS Negl. Trop. Dis. 5, e1160 (2011). 


\section{ONLINE METHODS}

Parasite material. Details of the origin of the P. cynomolgi $\mathrm{B}$, Berok and Cambodian strains, their growth in macaques and isolation of parasite material are given in the Supplementary Note.

Genome sequencing and assembly. P. cynomolgi B strain was sequenced using the Roche 454 GS FLX (Titanium) and Illumina/Solexa Genome Analyzer IIx platforms to $161 \times$ coverage. In addition, 2,784 clones $(6.8 \mathrm{Mb})$ of a $\sim 40-\mathrm{kb}$ insert fosmid library in pCC1FOS (EpiCentre Biotechnologies) was sequenced by the Sanger method. A draft assembly of strain B was constructed using a combination of automated assembly and manual gap closure. We first generated de novo contigs by assembling Roche 454 reads using GS De novo Assembler version 2.0 with default parameters. Contigs of $>500$ bp were mapped to the P. vivax Salvador I reference assembly ${ }^{12}$ (PlasmoDB; see URLs). P. cynomolgi contigs were iteratively arrayed through alignment to $P$. vivax-assembled sequences with manual corrections. A total of 1,264 aligned contigs were validated by mapping paired-end reads from fosmid clones using blastn $(e<1 \times$ $10^{-15}$; identity $>90 \%$; coverage $>200 \mathrm{bp}$ ) implemented in GenomeMatcher software version 1.65 (ref. 28). Additional linkages (699 regions) were made using PCR across the intervening sequence gaps with primers designed from neighboring contigs. The length of sequence gaps was estimated from insert lengths of the fosmid paired-end reads, the size of PCR products and homologous sequences of the $P$. vivax genome. Supercontigs were then manually constructed from the aligned contigs. Eventually, we obtained 14 supercontigs corresponding to the 14 chromosomes of the parasite, with a total length of $\sim 22.73 \mathrm{Mb}$, encompassing $\sim 80 \%$ of the predicted P. cynomolgi genome. A total of 1,651 contigs ( $>1 \mathrm{~kb}$ ) with a total length of $3.45 \mathrm{Mb}$ was identified as unassigned subtelomeric sequences by searching against the $P$. vivax genome using blastn. Additionally, to improve sequence accuracy, we constructed a mapping assembly of Illumina paired-end reads and the 14 supercontigs and unassigned contigs as reference sequences using CLC Genomics Workbench version 3.0 with default settings (CLC Bio). Comparison of the draft P. cynomolgi B sequence with 23 P. cynomolgi protein-coding genes $(64 \mathrm{~kb})$ obtained by Sanger sequencing showed $99.8 \%$ sequence identity (Supplementary Table 13). The P. cynomolgi Berok and Cambodian strains were sequenced to $26 \times$ and $17 \times$ coverage, respectively, using the Roche 454 GS FLX platform, with single-end and $3-\mathrm{kb}$ paired-end libraries made for the former and a single-end library only made for the latter. For phylogenetic analyses of specific genes, sequences were independently verified by Sanger sequencing (Supplementary Table 14 and Supplementary Note).

Prediction and annotation of genes. Gene prediction for the 14 supercontigs and 1,651 unassigned contigs was performed using the MAKER genome annotation pipeline $\mathrm{e}^{29}$ with $a b$ initio gene prediction programs trained on proteins and ESTs from PlasmoDB Build 7.1. For gene annotation, blastn $\left(e<1 \times 10^{-15}\right.$; identity $>70 \%$; coverage $>100 \mathrm{bp}$ ) searches of $P$. vivax (PvivaxAnnotated Transcripts_PlasmoDB-7.1.fasta) and P. knowlesi (PknowlesiAnnotatedTran scripts_PlasmoDB-7.1.fasta) predicted proteomes were run, and the best hits were identified. All predicted genes were manually inspected at least twice for gene structure and functional annotation, and orthologous relationships between P. cynomolgi, P. vivax and P. knowlesi were determined on synteny. A unique identifier, PCYB_\#\#\#\#\#, was assigned to P. cynomolgi genes, where the first two of the six numbers indicate chromosome number. Paralogs of genes that seemed to be specific to either $P$. cynomolgi, $P$. vivax or $P$. knowlesi were searched using blastp with default parameters, using a cutoff $e$ value of $1 \times 10^{-16}$.

Multiple genome sequence alignment. Predicted proteins of $P$. cynomolgi $B$ strain were concatenated and aligned with those from the 14 chromosomes of 5 other Plasmodium genomes: P. vivax, P. knowlesi, P. falciparum, P. berghei and $P$. chabaudi, using Murasaki software version 1.68.6 (ref. 30).

Search for sequence showing high similarity to host proteins. Eleven $P$. cynomolgi CYIR proteins (with sequence similarity to $P$. knowlesi KIR) were subjected to blastp search for regions having high similarity to host Macacca mulatta CD99 protein, with cutoff $e$ value of $1 \times 10^{-12}$ and compositional adjustment (no adjustment) against the nonredundant protein sequence data set of the M. mulatta proteome in NCBI.
Phylogenetic analyses. Genes were aligned using ClustalW version 2.0.10 (ref. 31) with manual corrections, and unambiguously aligned sites were selected for phylogenetic analyses. Maximum-likelihood phylogenetic trees were constructed using PROML programs in PHYLIP version 3.69 (ref. 32) under the Jones-Taylor-Thornton (JTT) amino-acid substitution model. To take the evolutionary rate heterogeneity across sites into consideration, the $\mathrm{R}$ (hidden Markov model rates) option was set for discrete $\gamma$ distribution, with eight categories for approximating the site-rate distribution. CODEML programs in PAML 4.4 (ref. 33) were used for estimating the $\gamma$ shape parameter, $\alpha$ values. For bootstrap analyses, SEQBOOT and CONSENSE programs in PHYLIP were applied.

Candidate genes for hypnozoite formation. We undertook two approaches. First, genes unique to P. vivax and P. cynomolgi (hypnozoite-forming parasites) and not found in other non-hypnozoite-forming Plasmodium species were identified. We used the 147 unique genes identified in the $P$. vivax genome ${ }^{12}$ to search the P. cynomolgi B sequence. For the orthologs identified in both species, $\sim 1 \mathrm{~kb}$ of sequence $5^{\prime}$ to the coding sequence was searched for four specific ApiAP2 motifs ${ }^{20}$ _PF14 0633, GCATGC; PF13_0235_D1, GCCCCG; PFF0670w_D1, TAAGCC; and PFD0985w_D2, TGTTAC-which are involved in sporozoite stage-specific regulation and expression (corresponding to the pre-hypnozoite stage). Second, dormancy-related proteins were retrieved from GenBank and used to search for $P$. vivax homologs. Candidate genes $(n=128)$ and orthologs of $P$. cynomolgi and five other parasite species were searched in the region $\sim 1 \mathrm{~kb}$ upstream of the coding sequence for the presence of the four ApiAP2 motifs. Data for P. vivax, P. knowlesi, P. falciparum, P. berghei, Plasmodium chabaudi and Plasmodium yoelii were retrieved from PlasmoDB Build 7.1.

Genome-wide screen for polymorphisms. For SNP identification, alignment of Roche 454 data from strains B, Berok and Cambodian was performed using SSAHA2 (ref. 34), with 0.1 mismatch rate and only unique matches reported. Potential duplicate reads generated during PCR amplification were removed, so that when multiple reads mapped at identical coordinates, only the reads with the highest mapping quality were retained. We used a statistical method ${ }^{35}$ implemented in SAMtools version 0.1.18 to call SNPs simultaneously in the case of duplicate runs of the same strain. SNPs with high read depth $(>100)$ were filtered out, as were SNPs in poor alignment regions at the ends of chromosomes (Supplementary Note).

Nucleotide diversity $(\pi)$ was calculated as follows. For each site being compared, we calculated allele frequency by counting the two alleles and measured the proportion of nucleotide differences. Letting $\pi$ be the genetic distance between allele $i$ and allele $j$, then the nucleotide diversity within the population is

$$
\pi=\sum_{i, j} P_{i} P_{j} \pi_{i j}
$$

where $P_{i}$ and $P_{j}$ are the overall allele frequencies of $i$ and $j$, respectively. Mean $\pi$ was calculated by averaging over sites, weighting each by $\sum_{i=1}^{n-1} \frac{1}{i}$ where $n$ is the number of aligned sites. Average $d_{N} / d_{S}$ ratios were estimated using the modified Nei-Gojobori/Jukes-Cantor method in MEGA 4 (ref. 36).

CNV-seq ${ }^{23}$ was used to identify potential CNVs in P. cynomolgi. Briefly, this method is based on a statistical model that allows confidence assessment of observed copy-number ratios from next-generation sequencing data. Roche 454 sequences from $P$. cynomolgi strain B assembly were used as the reference genome, and the $P$. cynomolgi Berok strain was used as a test genome; the sequence coverage of the Cambodian strain was considered too low for analysis. The test reads were mapped to the reference genome, and CNVs were detected by computing the number of reads for each test strain in a sliding window. The validity of the observed ratios was assessed by the computation of a probability of a random occurrence, given no copynumber variation.

Polymorphic microsatellites (defined as repeat units of 1-6 nucleotides) between P. cynomolgi strains B and Berok were identified by aligning contigs 
from a de nova assembly of Berok (generated using Roche GS Assembler version 2.6, with 40-bp minimum overlap, $90 \%$ identity) to the B strain using the Burrows-Wheeler Aligner (BWA) ${ }^{37}$ and allowing for gaps. Using the Predscaled probability of the base being misaligned by SAMtools ${ }^{35}$, indel candidates were called from the alignment. In-house Python scripts were used to then cross-reference with the microsatellites found in the reference strain B assembly identified by MISA (see URLs). All homopolymer microsatellites were discarded to account for potential sequence errors introduced by 454 sequencing.

Selective constraint analysis of 4,563 orthologs between P. cynomolgi strains B and Berok and 4,601 orthologs between these strains and P. viva Salvador I used MUSCLE ${ }^{38}$ alignments with stringent removal of gaps and missing data (P. cynomolgi Berok orthologs were identified through a reciprocal best-hit BLAST search against strain B genes). Analyses were conducted using the NiGojobori model ${ }^{25}$. To detect values that could not be explained by chance, we estimated the standard error by a bootstrap procedure with 200 pseudoreplicates for each gene. The expected value for $d_{S} / d_{N}$ is 0 if a given pair of sequences is diverging without obvious effects on fitness. In the case of the comparison within $P$. cynomolgi, values with a difference of \pm 2 s.e.m. from 0 were considere indicative of an excess of synonymous $\left(d_{S} / d_{N}>0\right)$ or nonsynonymous $\left(\mathrm{d}_{\mathrm{S}} / \mathrm{d}_{\mathrm{N}}<0\right)$ changes. In the case of the comparison between $P$. cynomolgi and $P$. viva, we used a more stringent criterion of \pm 3 s.e.m. from 0 .
28. Ohtsubo, Y., Ikeda-Ohtsubo, W., Nagata, Y. \& Tsuda, M. GenomeMatcher: a graphical user interface for DNA sequence comparison. BMC Bioinformatics 9, 376 (2008).

29. Cantarel, B.L. et al. MAKER: an easy-to-use annotation pipeline designed for emerging model organism genomes. Genome Res. 18, 188-196 (2008).

30. Popendorf, K., Tsuyoshi, H., Osana, Y. \& Sakakibara, Y. Murasaki: a fast, parallelizable algorithm to find anchors from multiple genomes. PLOS ONE $\mathbf{5}$, e12651 (2010).

31. Thompson, J.D., Higgins, D.G. \& Gibson, T.J. CLUSTAL W: improving the sensitivity of progressive multiple sequence alignment through sequence weighting, position-specific gap penalties and weight matrix choice. Nucleic Acids Res. 22, 4673-4680 (1994).

32. Felsenstein, J. PHYLIP, Phylogeny Inference Package, 3.6a3 en (University of Washington, Seattle, 2005).

33. Yang, Z. PAML 4: phylogenetic analysis by maximum likelihood. Mol. Biol. Evol. 24, 1586-1591 (2007).

34. Ning, Z., Cox, A.J. \& Mullikin, J.C. SSAHA: a fast search method for large DNA databases. Genome Res. 11, 1725-1729 (2001).

35. Li, H. A statistical framework for SNP calling, mutation discovery, association mapping and population genetical parameter estimation from sequencing data. Bioinformatics 27, 2987-2993 (2011).

36. Tamura, K., Dudley, J., Nee, M. \& Kumar, S. MEGA4: Molecular Evolutionary Genetics Analysis (MEGA) software version 4.0. Mol. Biol. Evol. 24, 1596-1599 (2007).

37. Li, H. \& Durbin, R. Fast and accurate short read alignment with Burrows-Wheeler transform. Bioinformatics 25, 1754-1760 (2009).

38. Edgar, R.C. MUSCLE: multiple sequence alignment with high accuracy and high throughput. Nucleic Acids Res. 32, 1792-1797 (2004).

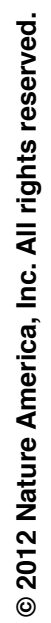

이 이

doi:10.1038/ng.2375

NATURE GENETICS 